\title{
La Macédoine des Jeunes-Turcs et l'hellénisme à travers la presse grecque : 1908-1910
}

Hellenism and the Young Turks Macedonia through the Greek newspapers 1908-1910

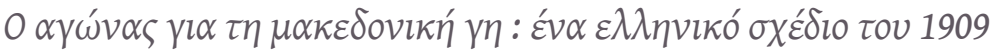

\section{Sia Anagnostopoulou}

\section{OpenEdition}

\section{Journals}

Édition électronique

URL : https://journals.openedition.org/ceb/1128

DOI : $10.4000 /$ ceb. 1128

ISSN : 2261-4184

Éditeur

INALCO

Édition imprimée

Date de publication : 9 janvier 2012

ISSN : 0290-7402

\section{Référence électronique}

Sia Anagnostopoulou, «La Macédoine des Jeunes-Turcs et l'hellénisme à travers la presse grecque 1908-1910 », Cahiers balkaniques [En ligne], 40 | 2012, mis en ligne le 25 mai 2012, consulté le 06 juillet 2021. URL : http://journals.openedition.org/ceb/1128; DOI : https://doi.org/10.4000/ceb.1128

Ce document a été généré automatiquement le 6 juillet 2021

\section{cc) (1) 8}

Cahiers balkaniques est mis à disposition selon les termes de la Licence Creative Commons Attribution - Pas d'Utilisation Commerciale 4.0 International. 


\title{
La Macédoine des Jeunes-Turcs et l'hellénisme à travers la presse grecque : 1908-1910
}

\author{
Hellenism and the Young Turks Macedonia through the Greek newspapers
}

1908-1910

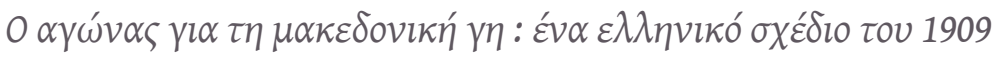

\section{Sia Anagnostopoulou}

Dans cet article, nous présenterons l'accueil que la presse grecque a réservé à la Révolution des Jeunes-Turcs, notamment pendant les premiers mois qui suivirent son éclatement (1908), ainsi que les fluctuations de sa position envers la politique des Jeunes-Turcs, de 1908 jusqu'à 1910. L'intérêt avec lequel la presse grecque suit les événements de 1908 est proportionnel à l'intérêt général en Grèce pour la Macédoine, la question nationale par excellence de l'époque. Ainsi, il nous intéresse d'étudier la réaction de la presse grecque envers la Révolution de 1908, à la lumière toutefois de la question macédonienne. Quelles sont, selon la presse, les conséquences de la Révolution sur la question macédonienne, sur les revendications grecques en général, telles qu'elles se forment dans le cadre de la Grande Idée? Ensuite, nous essayerons d'analyser les raisons alléguées par la presse pour justifier aussi bien l'enthousiasme pour la Révolution, que le changement de sa position envers les Jeunes-Turcs, quelques mois seulement après la Révolution. Nous nous limiterons à la première période du gouvernement des Jeunes-Turcs, plus précisément, la période qui s'étend entre 1908 et 1910, depuis donc la Révolution de 1908 et jusqu'à l'avènement au pouvoir en Grèce du Premier ministre Eleftherios Venizélos.

2 Cette période, où prédomine une certaine fluidité concernant la question macédonienne, est pour nous très importante. La politique grecque est encore confuse vis-à-vis de la Macédoine; la "nation grecque non délivrée " y est difficilement délimitée, les territoires revendiqués ${ }^{1}$ encore plus. D'autre part, la politique des JeunesTurcs n'est pas pendant cette période très claire, non seulement à propos de la 
Macédoine, mais plus généralement à propos de la nouvelle «identité » de l'Empire; le contenu des termes, tels que "millet ottoman», "patrie ottomane», étant difficilement défini et ouvert à plusieurs interprétations. Dans le cadre donc de cette fluidité, il est important, à notre avis, de suivre, à travers la presse grecque, les fluctuations des rapports entre les Jeunes-Turcs et la Grèce, à cause précisément de la Macédoine. Ces fluctuations toutefois découlent des modifications parallèles et interactives que le contenu de deux notions subit respectivement en Grèce et dans l'Empire : celui de la notion de la « nation grecque non délivrée » et celui de la notion $\mathrm{du}$ «millet des Rums». Nous constaterons que la Macédoine constitue l'exemple par excellence de la Question d'Orient, où non seulement les revendications territoriales antagonistes entre les pays balkaniques sont exprimées, mais où plusieurs programmes nationaux et modernistes, contradictoires et interactifs en même temps, se sont entrecroisés.

\section{La pluralité de versions de la Grande Idée : I'helléno- ottomanisme}

3 À l'aube de la révolution jeune-turque, la Macédoine constitue pour la Grèce la question nationale la plus importante et en même temps la plus inquiétante, aussi bien à cause de l'identité macédonienne propagée par la $\mathrm{VMRO}^{2}$, qu'à cause de l'action des bandes armées bulgares ${ }^{3}$. La prépondérance de l'élément bulgaro-slave surtout dans les campagnes, même aux alentours de Thessalonique, que les activistes, nationalistes grecs, ont essayé d'affronter depuis déjà quelques décennies ${ }^{4}$, demeure, encore en 1908 , un problème qui met en danger les revendications nationales grecques en Macédoine. Les programmes d'hellénisation de la population macédonienne se suivent, sans pour autant obtenir les résultats attendus ${ }^{5}$. En 1908 donc, devant le danger de bulgarisation ou de macédonisation de la Macédoine, la Grèce préfère promouvoir au niveau officiel l'idée de l'helléno-ottomanisme, et ceci en parallèle avec l'action sur place des bandes armées, agissant dans la région depuis 1903. L'idée de l'helléno-ottomanisme, présente depuis les dernières décennies du XIX ${ }^{e}$ siècle ${ }^{6}$, déguise la Grande Idée, ou, pour le dire différemment, constitue la voie alternative pour sa réalisation. Cette idée est plutôt le produit d'un opportunisme très caractéristique de la Grande Idée. L'expansion slave en Macédoine et la faiblesse grecque face à elle, à la fin du XIX ${ }^{e}$ siècle et début du XX siècle imposent de dissimuler la Grande Idée derrière un plan impérial selon lequel la cogestion de l'Empire et de ses territoires par ses deux éléments importants - Grec et Ottoman - est absolument nécessaire pour la sauvegarde de l'Empire, un empire helléno-ottoman et par suite hellénique. En 1880 Athanassios Paploukas-Eftaxias ${ }^{7}$, un des nationalistes, activistes grecs, note ainsi avec un cynisme sans détours :

"Le premier pas de l'hellénisme pour se rapprocher des musulmans [en Macédoine] est de s'exprimer sans réserve en faveur du régime [ottoman], [...]. C'est la seule politique prudente pour lui [l'hellénisme]. [...]. [Parce que] quand le musulman sera, avec le temps, familiarisé avec le Grec, quand il sera convaincu que celui-ci [le Grec] a respecté ses droits, qui est inévitable - quand il sera obligé de choisir entre le bulgarisme et l'hellénisme, il préférera ce dernier ; le musulman avec sa majorité écrasante aurait ainsi consolidé la place de l'hellénisme dans le pays [la Macédoine]"

4 Toutefois, cette idée de la collaboration entre l'élément turc et l'élément grec constitue en même temps, et de façon paradoxale, un élément structurel du nationalisme grec. En effet, derrière cette idée helléno-ottomane, se cache une conception orientaliste 
d'après laquelle la place dans le monde civilisé réservée aux Ottomans, aux Turcs en général, dépend de leur fonction en tant que force auxiliaire de l'hellénisme contre ses ennemis. Le Turc, incapable de développer une conscience nationale, incapable donc de revendications nationales ${ }^{8}$, ne peut être doté, toujours d'après les nationalistes grecs, que d'un seul rôle historique, qui va lui permettre son entrée dans le monde civilisé : celui de barrière contre le bulgaro-slavisme. L'hellénisme est, selon cette conception orientaliste, la seule nation civilisée et « occidentale » de la région, se battant toujours contre la barbarie des «autres». "Bien entendu les bulgarophones sont des brutes, mais des brutes qui parlent le bulgare et qui pourront, avec le soutien des autres, acheter un jour la terre [macédonienne], [...] », souligne avec une sensibilité remarquable Ion Dragoumis, un de plus fervents nationalistes Grecs, dans son mémorandum $(4 / 12 / 1903)^{9}$ destiné au ministère grec des Affaires étrangères.

5 L'helléno-ottomanisme prend de l'ampleur et se généralise lors de la Révolution des Jeunes-Turcs en 1908. Ainsi, le changement du régime dans l'Empire est accueilli à Athènes, avec enthousiasme et avec l'espoir de la réalisation de la souveraineté commune gréco-turque en Macédoine aussi bien par le peuple ${ }^{10}$ que par les journaux (Acropolis $^{11}$, Skrip ${ }^{12}$, Embros $^{13}$ ), comme d'ailleurs dans la Salonique ottomane par l'hebdomadaire socialiste, Rizospastis ${ }^{14}$. Tout d'abord, le changement de régime promis par la Révolution constitue pour la presse la barrière la plus efficace contre l'intervention des "étrangers» dans la question macédonienne. Le programme des réformes de Mürzsteg, décidé à l'unanimité par les Grandes Puissances après la révolte d'Iliden, inquiète la Grèce qui voit dans ces réformes un moyen de stabiliser le statu quo en Macédoine ${ }^{15}$; un statu quo nuisible pour ses intérêts. En effet, la Grèce, presque isolée diplomatiquement à cause, entre autres, de l'action de ses bandes armées en Macédoine ${ }^{16}$, a plusieurs raisons de s'inquiéter de cette intervention « des étrangers ».

6 La Révolution de 1908 est donc mise en avant comme l'arme offerte par la conjoncture pour servir la vision de la Grande Idée dans sa dimension helléno-ottomane, écrit un article du Rizospastis sous le titre, très caractéristique, «Aide tombée du ciel $»^{17}$ :

"Les Osmanli, étant lâches et désespérés, sortent maintenant de leur léthargie et ils anéantissent les rêves de ceux qui, sous le manteau de la diplomatie, agissaient en complicité avec les bandes, soi-disant autonomistes, en Macédoine. [...] "

7 Les autres journaux partagent aussi la même opinion et contribuent à créer un climat d'euphorie à propos de la Macédoine. Ainsi, selon le journal Acropolis du 17 juillet 1908 :

«Les Grandes Puissances n'ont aucune raison, sous prétexte de l'application des réformes, de se mêler désormais de la question macédonienne. La situation qui prédomine actuellement en Macédoine prouve que la fraternité entre les ethnies est, grâce à la proclamation de la liberté constitutionnelle, établie $»^{18}$.

8 Toutefois cette euphorie ne s'explique pas seulement par l'éloignement éventuel du danger « étranger » en Macédoine, mais surtout par l'espoir que la Question d'Orient en général, la question de la Macédoine plus précisément, sera résolue par la collaboration des deux éléments puissants de l'Empire, les Grecs et les Turcs. Le journal Acropolis, du 6 septembre 1908 explique :

"Notre joie et notre proturcisme dérivent, d'une part, du fait que le danger immédiat présageant la concession de la Macédoine aux Bulgares par les deux Puissances partiales, l'Angleterre et la Russie, est éloigné. D'autre part, ils dérivent du fait que l'idée de l'hellénoturcisme contre l'ennemi commun, le panslavisme, soit, au sein du nouveau régime, réalisée $»^{19}$. 
D'ailleurs, « l'Empire ottoman ne sera puissant que s'il sert de façon efficace les intérêts communs aux Grecs et aux Turcs ", souligne le même journal, argumentant ainsi sur sa position envers le nouveau régime ${ }^{20}$.

10 Toutefois, l'enthousiasme exprimé par les journaux grecs lors de l'éclatement de la Révolution ne doit pas être expliqué par l'helléno-ottomanisme seulement. Derrière l'helléno-ottomanisme se cache une admiration, tantôt ouverte, tantôt retenue. Les Grecs sont impressionnés par la Révolution: le Turc, le «barbare » d'hier, laisse sa place au Turc, capable de revendiquer un régime constitutionnel, un régime civilisé, et ceci sans l'aide des Grandes Puissances. La Révolution de 1908 rend d'un autre côté les Grecs perplexes, au moins dans la mesure où les journaux nous permettent de capter cette perplexité. En effet, les journaux, d'une part, utilisent la Révolution comme exemple, digne d'être imité par les Grecs dont le système politique, criblé de corruption, laisse beaucoup à désirer : «Il nous faut, à nous aussi, des Jeunes-Turcs. [...] nous devons travailler comme les Jeunes-Turcs pour sauver notre patrie et pour nous sauver de la pourriture, politique et gouvernementale » trouve-t-on dans l'article titré «Il nous faut, a nous aussi, des Jeunes-Turcs ${ }^{21}$. D'autres journaux, Rizospastis par exemple, ne manquent pas de faire des comparaisons entre les deux pays :

"Voilà l'image que le pays voisin donne maintenant, un pays qui était condamné à la décomposition à cause des abominations des Vieux Turcs [...]. Les Vieux Grecs conservateurs (oligarchiques et capitalistes) du passé doivent être, eux aussi, renversés de manière radicale par les Jeunes Grecs - progressistes - libéraux [...] pour le progrès national, politique et social "22. D'autre part pourtant, ils n'hésitent pas à exprimer quelques réticences à propos de la capacité des Turcs pour un tel changement politique. Les journaux grecs, piégés entre un nationalisme à nuance orientaliste, profondément ancré dans la conscience grecque, et l'embarras dans lequel les met la coexistence du Califat et du Sultanat avec la Constitution, s'interrogent sur les messages secrets de cette Révolution: "le plus grand problème est le suivant. Comment les relations entre conquérant et conquis seront-elles abolies? Comment la Conquête sera-t-elle oubliée ? Comment la vraie égalité politique serat-elle réalisée, puisque le Turc vise la Charya et les fetva et puisque la loi sainte écarte toute possibilité d'égalité entre eux [musulmans] et chrétiens? [...]. Nous n'oublions pas que le sultan n'est pas un simple souverain, il est en même temps le calife. Et si le Sultanat était en harmonie avec le Califat au sein de la monarchie, ils seront en lutte au sein du système constitutionnel $»^{23}$.

11 Néanmoins, la Révolution offre à la presse grecque, même la plus conservatrice, la base qui légitime la propagation de l'helléno-ottomanisme. En effet si l'helléno-ottomanisme constituait avant la Révolution un choix obligatoire et stratégique, limité à l'entourage fermé des élites politiques, mais difficilement justifiable auprès du peuple en tant que politique nationale, la situation change à l'occasion de la Révolution. L'hellénoottomanisme commence à se propager dans le grand public, à travers les journaux, comme expression des revendications nationales ; l'ennemi national d'hier peut être le sauveur national d'aujourd'hui. Une nouvelle conception nationale, ou plutôt, un nouveau stéréotype est en train, à travers la presse, de se former, un stéréotype - qui sera d'une grande longévité - selon laquelle l'opposition entre les Grecs et les Turcs résulte de l'intervention des Grandes Puissances :

"Jusqu'à hier, les Grandes Puissances [...] cherchaient des moyens pour frapper efficacement et mortellement les Grecs et les Turcs, en créant de la brouille et de l'inimitié entre eux. Mais aujourd'hui, après la proclamation de la Constitution [...] l'Empire est réformé entièrement [...]. [...] ces réformes générales et radicales [...], ont remplacé les [réformes] limitées et partiales à travers lesquelles [les Grandes Puissances] préparaient l'annexion des provinces, auxquelles ces réformes seraient appliquées, à des pays étrangers [...]. Aujourd'hui la 
question macédonienne n'existe plus, parce que [l'Empire] a pris soin de la Question d'Orient dans son ensemble $[. ..] \|^{24}$.

La Révolution est considérée comme la preuve par excellence qui légitime l'hellénoottomanisme, et la presse grecque ne se permet aucune critique contre elle. La façon avec laquelle la presse affronte l'opposition du Patriarcat de Constantinople à la Révolution et au rétablissement de la Constitution ${ }^{25}$ est caractéristique, elle varie entre la critique ouverte et sévère et la critique d'arrière-plan sous la forme de conclusion rassurante. Le journal Acropolis, dans l'article au titre très explicite Les inquiétudes $d u$ Patriarche, exprime sa position sous-jacente, selon laquelle les autorités religieuses ne doivent pas avoir un rôle politique au sein d'un système constitutionnel :
"Déclarations inouies du Patriarche $»^{26}$, critique sévèrement ce dernier qui a osé exprimer au grand vizir sa consternation pour le rétablissement de la Constitution; le journal considérant les inquiétudes exprimées par le Patriarche dénuées de fondement explique que: "[...] le pouvoir politique du Patriarche sera limité, mais son pouvoir spirituel sera étendu $»^{27}$

13 Toutefois pour la majorité de la presse grecque, notamment la plus conservatrice ${ }^{28}$, l'helléno-ottomanisme comprend en préalable, le maintien, de la part des Jeunes-Turcs, des privilèges du Patriarche de Constantinople, privilèges qui constituent la preuve de droits historiques de l'hellénisme dans l'Empire ${ }^{29}$.

\section{L'helléno-ottomanisme, une des versions de l'ottomanisme}

Cette idée de l'helléno-ottomanisme cherche sa base légitimatrice dans une tradition helléno-ottomane, une continuité helléno-ottomane ininterrompue, justifiée par l'existence du Patriarcat de Constantinople et de ses privilèges, sous l'égide duquel tous les Orthodoxes - Bulgares, Grecs, Serbes, etc. - de la Macédoine (de l'Empire entier) se trouvaient. Constantinople, ottomane et orthodoxe en même temps, est le centre de la légitimation de l'unité macédonienne, de l'unité helléno-ottomane. Alors, si le remède à la question macédonienne est l'unité de tous les éléments (ittihad-i anasir) composant le millet ottoman, cette unité est - notamment selon les Grecs de l'Empire - assurée par les deux piliers de l'Empire :

"Le Califat et le Patriarcat sont les deux grandes autorités sous lesquelles la balance nationale de notre empire est fondée. [...]. En effet, quand l'Empire ottoman a été fondé le Califat est devenu l'établissement ottoman du monde musulman, alors que le Patriarcat a été nommé, suite aux droits et aux privilèges concédés par le Conquérant [...], l'établissement ottoman du monde chrétien. Aussi bien le Califat que le Patriarcat sont basés sur les mêmes principes salutaires, assurant l'unité religieuse et non pas nationale. Il y a dans l'empire des Arabes, des Slaves, des Albanais, des musulmans et des chrétiens, mais tous les Turcs sont des musulmans comme tous les Grecs sont des chrétiens. Par conséquent, ces sont les deux peuples qui, à travers le Califat et le Patriarcat [...], assurent l'unité [...] ottomane " ${ }^{30}$.

L'association, dans le discours grec, des institutions anciennes, tel que le Patriarcat de Constantinople (et ses privilèges), et du système constitutionnel (avec les notions d'égalité et de droits des peuples), n'est pas sans intérêt. Ce discours politique est propagé par les députés grecs-orthodoxes au parlement ottoman de Constantinople ${ }^{31}$, mais son centre de diffusion par l'intermédiaire de la presse, est Athènes. En effet, l'intérêt de la presse grecque, qui partage complètement ce raisonnement, se focalise sur la politique des Jeunes-Turcs envers les privilèges du Patriarcat. Son enthousiasme 
pour le changement politique dans l'Empire est en grande partie dû à la satisfaction ressentie face à la politique des Jeunes-Turcs envers le Patriarcat et ses privilèges ; même les journaux, tels qu'Embros ou Scrip, qui ont accueilli la Révolution avec une certaine réticence, ne cachent pas leur satisfaction. Le journal Embros ${ }^{32}$, résume ainsi les principes de la bonne gouvernance dans l'Empire :

"Si quelque chose, parmi ce qui est survenu ces derniers temps en Turquie, doit attirer notre attention, ce sont les déclarations faites par le Comité Union et Progrès concernant le maintien des privilèges de la Grande Église et du Génos (millet, dans le sens d'une nation grecque orthodoxe). Il n'y a rien de plus efficace de cette déclaration pour gagner la confiance de la nation grecque. Nous devons avouer que la diligence des Jeunes-Turcs à nous épargner toute inquiétude, démontre non seulement leurs intentions sincères pour la coexistence harmonieuse de tous les peuples en Turquie, mais leur prévoyance politique [...], n'ayant qu'un seul but, celui de faire la Turquie un pays fort. "

D'ailleurs, le journal Scrip explique clairement que, une fois les privilèges du Patriarcat respectés, le programme politique des Grecs est identique aux idées au nom desquelles s'effectue le changement dans l'Empire ${ }^{33}$.

Toutefois cette idée de l'helléno-ottomanisme propagée à Constantinople, bien qu'elle soit une version ottomane du nationalisme grec dont les racines se trouvent à Athènes, n'est pas dépourvue d'un certain patriotisme ottoman déclaré; mais la notion d'« ottoman" se vide de plus en plus de son contenu, non seulement pour les Grecsorthodoxes, mais pour les autres groupes ethniques de l'Empire - Jeunes-Turcs inclus. En d'autres mots, à travers l'helléno-ottomanisme propagé à Constantinople, certains points de la Grande Idée d'Athènes sont en voie d'ottomanisation, et ceci au nom de la Constitution. L'ottomanisme constitue désormais le chapeau commun, porté par chaque nationalisme s'activant au sein de l'Empire, qui revendique ainsi sa légitimité au nom de la tradition ottomane et de la Constitution ${ }^{34}$. Cette couverture d'ottomanisme est pour le moment nécessaire à toutes les nations de l'Empire, les antagonismes nationalistes notamment en Macédoine étant si violents que chaque nation a besoin de la protection de l'État ottoman, le seul à assurer l'unité du territoire macédonien, menacée par les adversaires.

En effet, si nous suivons les discussions qui ont lieu au parlement ottoman au début de 1909 à propos de la Macédoine, nous constatons qu'aussi bien les Grecs que les autres éléments ethniques de la région revendiquent leur patriotisme ottoman, en l'interprétant toutefois selon une tradition ottomane nationalisée, inventée, et en même temps modernisée dans la source constitutionnelle. Nous pourrions ainsi parler, en symétrie avec l'helléno-ottomanisme, d'un turco-ottomanisme des Jeunes-Turcs, un bulgaro-ottomanisme des Bulgares, comme d'ailleurs d'un arméno-ottomanisme des Arméniens $^{35}$, etc. ; chacun d'eux revendique l'unité ottomane de la Macédoine, justifiée toutefois au nom d'une lutte permanente pour la «modernité ottomane " que justement cette nation ottomane offrait contre l'adversaire qui minait la modernité, et, par conséquent l'unité ottomane. Les discussions au parlement ottoman sur la question macédonienne sont très révélatrices du contenu que chaque élément ethnique de l'Empire donne à la modernité ottomane. Ainsi, les députés bulgares légitiment au parlement l'action des bandes armées bulgares en Macédoine, au nom des principes de la liberté et d'égalité, qui sont par la suite proclamés par la Constitution. D’après leur raisonnement donc, le fait que les Bulgares se battent pour leur indépendance, ecclésiastique et éducative, face au Patriarcat de Constantinople, constitue la preuve par excellence de leur lutte contre l'ancien régime ${ }^{36}$, puisque la politique patriarcale en 
Macédoine est identifiée à l'ancien régime, à la tyrannie. Dans ce contexte, les Bulgares légitiment leur ottomanisme au nom d'un passé ottoman, interprété à la lumière de leur lutte permanente contre la tyrannie de l'ancien régime que le Patriarcat incarne pour les orthodoxes: «L'État ottoman n'est ni turc ni rum [dans le sens de l'orthodoxie patriarcale]. L'État ottoman ne sera ottoman que par les Ottomans. [...]. Les premiers qui ont lutté pour la liberté sont les Bulgares ", le député du Monastir, Pancedoref Efendi ${ }^{37}$, explique au parlement, justifiant ainsi le patriotisme bulgaro-ottoman qui s'est manifesté avant celui des Jeunes-Turcs. Dans ce cadre, l'intervention « des étrangers » en Macédoine est due au fait que ces derniers voyaient dans la lutte des Bulgares pour la liberté, une "modernité occidentale » revendiquée par l'un des éléments ottomans se battant contre la tyrannie.

19 Les députés grecs, par contre, qui accusent les Bulgares de quasi-trahison, puisque leur activité nationaliste en Macédoine a impliqué l'intervention des "étrangers » et a menacé l'intégrité du territoire ottoman, revendiquent une "modernité ottomane», conforme à la tradition ottomane - "notre méthode de consultation" (usulü Mesveretimize), d'après l'expression du député Kosmidi Efendi ${ }^{38}$; les Bulgares sont ainsi accusés de propager une « modernité étrangère et différentialiste ", et ils ont prouvé la même indifférence et manque de patriotisme que l'ancien régime ${ }^{39}$. Il est clair que les Grecs se présentent comme les dépositaires diachroniques d'un patriotisme ottoman, basé sur les deux principes par excellence de l'ottomanisme: Osmanlilik ve Rumluk, d'après l'expression du député Yorgi Boso (Georges Boussios) ${ }^{40}$. Toutefois, la notion de rumluk est, de la même façon que l'osmanlilik, vide de contenu, s'identifiant de plus en plus non pas à l'ensemble des orthodoxes, mais aux Grecs. Les bandes armées grecques donc ont lutté en Macédoine, au nom de l'ottomanisme qui avait, dans un passé lointain et glorieux, assuré l'unité de la Macédoine, du territoire ottoman en son ensemble, contre l'ancien régime et le nationalisme bulgare, ces deux derniers ayant abandonné les principes fondamentaux du patriotisme ottoman : l'osmanlilik et le rumluk. Osmanlilik ve rumluk constituent, d'après leur raisonnement, les piliers de la "modernité ottomane » dont le vecteur est l'État constitutionnel. Ainsi, l'action de bandes armées grecques dans la Macédoine est justifiée au nom de la liberté du peuple ottoman, contre ses ennemis : l'ancien régime et les Bulgares. De ce point de vue, les Grecs ont préparé en quelque sorte la Révolution de 1908.

Les Jeunes-Turcs par ailleurs marquent un nouveau point de départ du patriotisme ottoman, de l'ottomanisme : la Révolution effectuée par le Comité Union et Progrès. Dans ce cadre, il n'y a avant la Révolution aucune manifestation patriotique, aucune lutte pour la liberté, légitimée au nom de quelque élément ethnique que ce soit. Plus précisément pour la Macédoine, deux fronts ont été formés, l'un, antipatriotique, composé par les bandes armées, bulgares et grecques, et l'autre, patriotique, celui du peuple révolutionnaire, que le Comité allait exprimer: «[...], le Comité Union et Progrès a ouvert ses bras à tous les patriotes [révolutionnaires] et a arrêté l'activité des bandes armées", souligne le député Rahmi bey [Selanik] ${ }^{41}$ dans son discours au parlement. Il est caractéristique que, selon toujours le discours des députés JeunesTurcs, l'antipatriotisme des bandes armées en Macédoine soit dirigé contre le peuple, notamment contre les Turcs:

"Le peuple a été dégoûté [par l'action des bandes armées]. Tous ont commencé à être stupéfaits de la patience des Turcs $»^{42}$. L'identification de la notion du peuple avec la notion $d u$ Turc est déjà, bien que de façon implicite encore, en voie d'évolution. Ce qui est déjà clair pourtant, c'est le fait que la Révolution éclate au nom et après la demande du peuple 
ottoman, dont la volonté est exprimée par le Comité Union et Progrès. Dans ce cadre, les

Bulgares et les Grecs «ne travaillaient pas pour la liberté, mais pour l'absolutisme

[istibdat] $»^{43}$.

(a)

assurée par un gouvernement constitutionnel - mesrutiyeti idare. Le principe par excellence de ce gouvernement est la justice - adil. À partir de la notion adil, les Jeunes-Turcs inventent un passé ottoman, glorieux, où l'État ottoman devait sa puissance à la justice, à travers laquelle l'égalité de tous et la liberté de pratiquer sa religion étaient assurées. Cette justice traditionnelle ottomane, que l'ancien régime avait abandonnée, est rétablie par la Révolution de 1908 et la Constitution ${ }^{44}$. Le gouvernement constitutionnel donc, issu d'une Révolution menée par le Comité Union et Progrès au nom du peuple, est un gouvernement de justice, vecteur d'une modernité ottomane, européenne : « Aujourd'hui nous avons un gouvernement juste. L'Europe nous applaudit » le député Rahmi bey ${ }^{45}$ souligne au parlement. D'après donc le raisonnement des Jeunes-Turcs, la loyauté de toutes les ethnies à l'État constitutionnel ne peut être prouvée que par leurs dévotion et soumission à la politique du gouvernement, le seul qui exprime la volonté du peuple ottoman, du millet ottoman dans son ensemble. Toutefois, cet État constitutionnel est plutôt l'œuvre des Turcs musulmans, de ceux "qui ont subi les plus grands malheurs durant l'ancien régime " ${ }^{46}$. Dans ce cadre, les principes modernes, tels que le patriotisme, l'égalité et la liberté, ainsi que le droit de l'invention de la tradition ottomane, sont appropriés au nom du millet ottoman par le gouvernement des Jeunes-Turcs, le seul à avoir le droit légitime de reformuler le contenu de la notion ottomane; la revendication fragmentaire de l'ottomanisme constituant une trahison contre le millet ottoman.

\section{La fin de l'helléno-ottomanisme. Athènes, centre de l'Hellénisme}

Jusqu'aux premiers mois de 1909, les journaux grecs continuent à soutenir le gouvernement des Jeunes-Turcs, bien qu'ils commencent à douter sérieusement de son efficacité à consolider l'ottomanisme - à savoir l'helléno-ottomanisme - notamment en Macédoine, contre le bulgarisme. Dans ce cadre, la modernité du régime des JeunesTurcs et leur dévotion aux principes constitutionnels sont évaluées par la presse grecque en rapport avec leur capacité à protéger l'hellénisme en Macédoine contre les Bulgares. Ainsi, la presse grecque, ne s'interrogeant point sur l'action des bandes armées grecques en Macédoine - territoire d'un État constitutionnel - commence à s'interroger, à chaque occasion qui se présente, sur l'efficacité du constitutionnalisme des Jeunes-Turcs. Le journal Acropolis par exemple qui quelques mois seulement auparavant, accusait les Grecs de fanatisme contre les Bulgares, fanatisme qui mettait les Jeunes-Turcs en position difficile ${ }^{47}$, change en 1909 complètement sa ligne :

"Les Turcs effectuent des visites à domicile arbitraires chez les Grecs [...]. Les enquêtes sont inspirées par les Bulgares, les derniers [...] essayant d'accuser les Grecs de trahison. Cette collaboration entre Turcs et Bulgares est contre la Constitution $»^{48}$.

Cahiers balkaniques, $40 \mid 2012$ 

malheurs de l'hellénisme. Le journal Embros ${ }^{49}$ l'expose clairement en mai 1909:

\begin{abstract}
"Si nous comparons la situation accablante que le chauvinisme jeune turc crée maintenant en Macédoine avec celle de la période de l'absolutisme, nous constaterons que la différence ne fait pas honneur aux partisans de la Constitution. Le programme qu'ils appliquent, les idées qui les inspirent, ainsi que la politique d'affaiblissement de l'élément grec qu'ils adoptent, constituent des faits dont personne n'est en position de prévoir les conséquences ", Le journal Scrip, un mois plus tard, ajoute :

«Les Jeunes-Turcs n'ont aucune idée de la politique. Ils deviennent les organes des Bulgares, car, ils ne sont pas en mesure d'en saisir les machinations, aveuglés comme ils sont par leur haine des Grecs " $"$.
\end{abstract}

Toutefois, le changement de la position de la presse grecque envers les Jeunes-Turcs ne peut pas être justifié par le seul problème macédonien, car ce dernier s'intègre dans une vision de la Grande Idée que la politique des Jeunes-Turcs ne pouvait pas servir. En effet, quelques mois après la Révolution de 1908, notamment après la contre-révolution du 31 mars 1909 et le rétablissement des Jeunes-Turcs au pouvoir, les élites politiques grecques réalisent que ces derniers développent une politique de raison d'État, une politique centralisatrice, ayant pour but la sauvegarde de l'État ottoman, l'ottomanisation donc du territoire ottoman. La centralisation des pouvoirs, notamment en Macédoine où Mahmud Sevket Pacha - «l'abominable dictateur » ${ }^{51}$, selon les journaux grecs - est nommé inspecteur général d'Istanbul, de Monastir et d'Andrinople, a secoué les Grecs qui commencent à réaliser que la réottomanisation de la Macédoine implique la marginalisation de toute force agissant en dehors de l'État. Par ailleurs, l'ottomanisme des Jeunes-Turcs, à connotations nationalistes, surprend les Grecs pour lesquels la conscience nationale constitue une vertu réservée seulement aux nations civilisées, c'est-à-dire à la nation grecque, le Turc ne pouvant exprimer que du fanatisme. Dans ce contexte, le plan de l'helléno-ottomanisme avec centre de référence Istanbul se révèle inefficace, au moins pour la Macédoine. La presse grecque donc ne tarde pas à exprimer sa déception face à la politique des Jeunes-Turcs, ces derniers n'étant pas à la hauteur de ses rêves, à la hauteur donc du monde civilisé, européen et moderne, puisque l'hellénisme et ses rêves constituent la règle de l'européanisme, de la civilisation et de la modernisation des Jeunes-Turcs: "Vous vous trompez, si vous croyez que vous allez par la simple utilisation des mots Constitution, liberté et Assemblée persuader l'Europe que vous n'êtes pas les Turcs d'hier. Les Grecs souffrent et l'Europe vous voit ", écrit le journal Embros, le 2 mars 1909. Le rêve d'Istanbul, centre de réalisation de l'helléno-ottomanisme, est désormais abandonné; Athènes est le seul centre de l'hellénisme, la seule force politique, capable de le protéger (le Patriarcat y compris) et de négocier avec l'État ottoman en son nom.

«La politique de la Turquie - écrit Rizospastis - envers l'hellénisme, notamment envers les Grecs de la Macédoine, dissout les rêves de ceux qui ont cru que le changement du régime en Turquie suffisait pour que les intérêts des Grecs soient, sans l'intervention d'Athènes, protégés ${ }^{52}$.

Les journaux grecs explosent quand le gouvernement jeune turc applique la nouvelle loi pour l'éducation, qui prévoit le remplacement du système éducatif précédent par un système éducatif centralisé sous le contrôle direct de l'État ${ }^{53}$. Cette explosion toutefois arrive à son point culminant, lorsque le gouvernement ottoman impose le boycott des produits grecs dans tous les ports ottomans (Smyrne, Thessalonique, Istanbul, etc.) à cause de la question crétoise ${ }^{54}$. La presse grecque présente désormais tous les "problèmes nationaux ", la question macédonienne incluse, sous la même logique ; le 
Turc est l'ennemi éternel de l'hellénisme, en état de persécution permanente à cause du chauvinisme turc. Pour la première fois, l'hellénisme de la Grande Idée se dresse, à travers la presse, en chair et en os, en tant qu'un ensemble ${ }^{55}$ qui s'étend de l'Asie Mineure jusqu'à Crète, la Macédoine, l'Épire, etc. Désormais, il n'y a plus une question macédonienne, une question crétoise, des Grecs d'Asie Mineure, il n'y a que l'hellénisme en péril. Le Rizospastis, journal qui propage presque quotidiennement l'idée de la reconstruction de l'hellénisme autour d'Athènes exclusivement écrit ainsi en juin 1909 :

«La nouvelle Turquie constitutionnelle aspire à l'islamisation de tous les chrétiens et c'est ainsi qu'ils les terrorisent. Les Jeunes-Turcs, en commençant par les Grecs de la Macédoine, poussent l'hellénisme dans les bras d'Istanbul, les obligeant de rompre leurs relations avec Athènes $\|^{56}$

La presse grecque découvre désormais une nouvelle catégorie d'analyse de la politique des Jeunes-Turcs, celle du turcisme, une catégorie synonyme de la barbarie, innée chez les Turcs depuis l'éternité. Nous croyons assez intéressant de citer un extrait in extenso de la définition du turcisme, telle qu'elle est donnée par cette presse :

«Le nouveau Calife, Mehmed V, a juré - comme d'ailleurs avait juré son frère déchu - qu'il va conserver la Constitution et la Charya, deux choses pourtant inconciliables. La nature religieuse, politique et sociale du Turcisme ne tolère pas les libertés, les égalités et les fraternités. [Cette nature du turcisme] divise le genre humain en deux parties, complètement séparées l'une de l'autre, croyants et non-croyants. Il est impossible que les non-croyants, les giaours, soient [...] les égaux des croyants. Ils sont maudits par Alla [...] et il faut qu'ils soient les esclaves des croyants [...]. N'admirez pas, ne vous émouvez pas à l'idée que le Turcisme veut se baptiser dans la source des idées libérales. Soyez sûrs qu'il sortira encore plus crasseux qu'il y est entré. [...] La nature turque est le sable du désert, il est la terre sèche sur laquelle les épines de la tyrannie germent $»^{57}$.

La lune de miel est à jamais passée : les Turcs sont des barbares, inconditionnellement.

Ce changement radical de la position de la presse grecque reflète bien entendu le changement de la politique d'Athènes envers les Jeunes-Turcs. Athènes ne voit plus aucune raison de soutenir l'intégrité de la Macédoine, l'intégrité de l'Empire en général. Il est vrai que la politique grecque avait commencé, depuis 1906, à se militariser. La lutte en Macédoine et la révolte en Crète (1905) et, d'autre part, le renforcement économique des forces armées grecques ont contribué à cette militarisation $^{58}$. Par ailleurs, la société grecque, militarisée de plus en plus elle aussi, à cause d'une mobilité sociale difficilement contrôlée par les partis politiques ${ }^{59}$, soutient les choix militaires du gouvernement. Cette militarisation aussi bien du politique que du social se manifeste de façon claire et nette en août 1909, lors du coup militaire, connu sous le nom mouvement de Goudi, mené par un officier de liaison, auquel les commerçants et les petits-bourgeois d'Athènes avaient adhéré ${ }^{60}$. L'avènement au pouvoir d'Elefterios Venizélos en 1910 contribue à rationaliser cette militarisation de la Grèce, à rationaliser ses revendications nationales, aussi bien que l'opportunisme de la Grande Idée. Ainsi, l'helléno-ottomanisme que la presse grecque diffusait entre 1908 et début 1909, est abandonné, laissant la place à une Grande Idée centrée exclusivement autour d'Athènes, centre politique et militaire de l'ensemble de l'hellénisme, qu'il soit délivré ou non. Le pilier sur lequel repose cette nouvelle interprétation de la Grande Idée est encore une fois le Patriarcat de Constantinople et ses privilèges. Toutefois, le Patriarcat est, cette fois, appelé à multiplier et légitimer la politique d'Athènes au sein des Grecs orthodoxes comme auprès du gouvernement des Jeunes-Turcs; ses privilèges, rétrospectivement hellénisés, constituent désormais la preuve historique de l'existence 
d'une partie de l'hellénisme sous la domination turque ${ }^{61}$. De la même façon, à l'égard des autres nations chrétiennes sous domination turque, l'État constitutionnel ottoman s'est prouvé aussi tyrannique que celui de l'époque absolutiste, parce qu'il est incapable de respecter les droits nationaux des nations chrétiennes. Dans ce cadre, le terme ottoman est synonyme du terme tyrannie. Selon ce raisonnement :

" [...] dans ce pays, il n'y a qu'un seul État, l'état constitutionnel Ottoman [...], et plusieurs nations, [...], notamment chrétiennes. Par contre, il [le gouvernement] confond des termes [...] tels que 'Nation ottomane', 'État ottoman', 'Ethnie'. [...]; en ce qui concerne la 'Nation ottomane', il n'y a rien à dire parce que la 'Nation ottomane' est un terme de langage, mais en réalité elle n'existe pas " ${ }^{62}$. sort, commence à s'élaborer. L'opportunisme de la Grande Idée trouve ainsi une autre expression politique maintenant, celle de l'alliance balkanique.

\section{BIBLIOGRAPHIE}

Anagnostopoulou, Sia, (2010), “The 'Nation' of the Rum Sings of its Sultan: The many Faces of Ottomanism ”, in L. T. Baruh - V. Kechriotis (éd.), Economy and Society on Both Shores of the Aegean, Athènes.

Anagnostopoulou, Sia, (1997), Asie Mineure, 19 s.-1919. Les communautés grecques orthodoxes. Du millet de Rums à la nation grecque (en grec), Athènes : Ellinika Grammata.

Balta, Nasi, Despoina Papadimitriou, (1993), Notes sur l'histoire de la presse. La dimension grecque et européenne (en grec), « Odysseas », Athènes.

Bohotis, Athanasios, (1999), « Politique intérieure », dans L'Histoire de la Grèce au XX ${ }^{e}$ s., (en grec) v. I, IIe partie, 1900-1922. Les origines, Athènes : Vivliorama.

Dakin, Douglas, (1962), “The Greek Proposals for an Alliance with France and Great Britain, June July 1907", Balkan Studies, 3.

Gardikas-Katsiadakis, Helen, (1995), Greece and the Balkan Imbroglio: Greek Foreign Policy, 1911-1913, Athènes : Syllogos Pros Diadosin Ofelimon Vivlion.

Karakişla, Yavuz, (2008), Interview dans Istanbul Universitesi Siyasal Bilgiler Fakultesi Dergisi [Revue de la Faculté d'Études Politiques de l'Université d'Istanbul], n 38, mars 2008 (Numéro spécial sur la Révolution de 1908).

Karavas, Spyros, (2010), « Bienheureux ceux qui possèdent la terre ». Esquisses foncières pour l'expropriation de consciences en Macédoine, 1880-1909 (en grec), Athènes : Vivliorama.

Papaloukas-Eftaxias, Athanasios, (1880), L'œuvre de l'Hellénisme en Macédoine (en grec) Athènes : Typografion Vlastou.

Skopetea, Elli, (1999), « Les Grecs et ses ennemis. La situation de la nation au début du $20^{\mathrm{e}} \mathrm{s}$. », dans L'Histoire de la Grèce au XXe siècle., v. I, IIe partie, 1900-1922. Les origines (en grec)

Athènes :Vivliorama.

Souliotis-Nikolaidis, Athanasios, L'Organisation de Constantinople, (en grec), Athènes. 


\section{NOTES}

1. Pour la politique grecque en Macédoine pendant cette période, cf. l'excellent ouvrage de

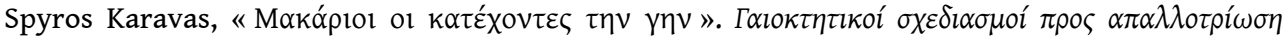

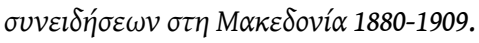

(«Bienheureux ceux qui possèdent la terre ». Esquisses foncières pour l'expropriation de consciences en Macédoine, 1880-1909), Athènes : Vivliorama, 2010, notamment pp. 172-229.

2. La VMRO (Organisation Autonomiste et Révolutionnaire de la Macédoine), fondée en 1893 , propage une nouvelle identité, celle du Macédonien et propulse un programme national - la Macédoine aux Macédoniens - et social - la libération sociale de la tyrannie des grands propriétaires fonciers. Les activités de la VMRO inquiètent la Grèce qui voit derrière cette organisation les Bulgares; l'identité «macédonienne » étant considérée comme l'équivalant de l'identité bulgare.

3. La Grèce est très tardivement intervenue dans l'affaire macédonienne, et ceci à cause de la " guerre malheureuse » de 1897 contre l'Empire ottoman. Les premières bandes armées arrivent en Macédoine en 1903 sous la direction du Comité macédonien, fondé à Athènes la même année. Le Comité prend de l'ampleur en 1904, lors de la mort d'un jeune officier - Pavlos Mélas - qui meurt en Macédoine-Occidentale se battant contre les Bulgares. Sa mort provoque une émotion panhellénique et la «lutte macédonienne » prend les dimensions de la cause nationale par excellence. Après quatre ans (1904-1908) de guerre, dure et sanglante, les bandes grecques ne réussissent pas à résoudre le problème macédonien ; l'hellénisation de la Macédoine demeurant un but éloigné.

4. Pour la situation ethnologique de la Macédoine et le choc que celle-ci provoque chez les nationalistes qui agissent sur place, voir, le rapport de l'activiste Athanasios Papaloukas-Eftaxias,

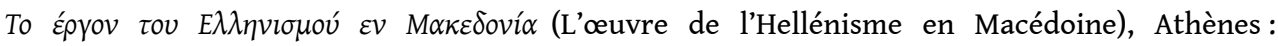
Typografion Vlastou, 1880, pp. 15-20, publié par Karavas, op. cit, notamment pp. 261-267.

5. Pour une analyse originale de ces projets, voir, Karavas, op. cit, où les programmes de l'hellénisation de la Macédoine, proposés par les nationalistes Grecs, sont donnés en annexe.

6. Pour l'helléno-ottomanisme, voir, Sia Anagnostopoulou, “The 'Nation' of the Rum Sings of its Sultan: The many Faces of Ottomanism ", in L. T. Baruh - V. Kechriotis (éd.), Economy and Society on Both Shores of the Aegean, Athènes, 2010, pp. 79-105.

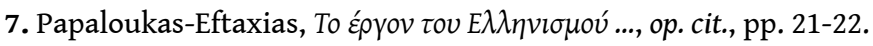

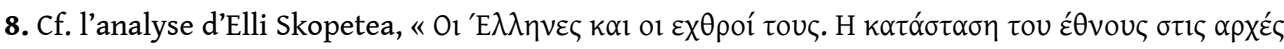

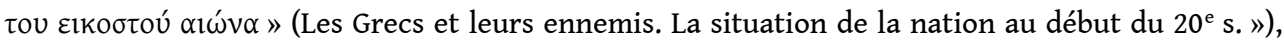

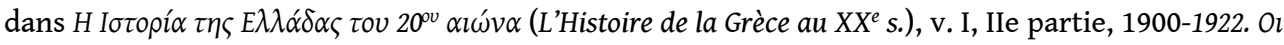
$\alpha \pi \alpha \rho \chi \varepsilon ́ \varsigma$ (1900-1922 Les origines), Athènes : Vivliorama, pp. 9-35, pp. 12-14.

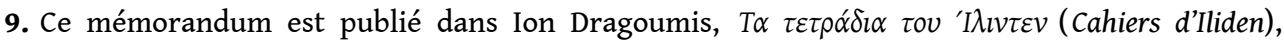
pp. 632-634.

10. Dans les rues d'Athènes, plusieurs manifestations ont lieu en faveur de la Révolution. Les manifestants déclarent ouvertement que les Grecs et Turcs partagent un sort commun et cette déclaration ne semble «scandaliser personne », comme Elli Scopetea le note pertinemment, op. cit, p. 14.

11. Acropolis, «le plus ancien journal grec », d'après la légende qui figure sur la première page du journal, a été publié le 30 octobre 1883, par Vlassis Gabriilides, journaliste istanbuliote, déporté par le sultan à cause de la combativité de ses articles. La diffusion du journal a été interrompue en 1920, lors de la mort de son fondateur. Il paraît de nouveau en 1929, sous la direction de G. Voutsinas. L'Acropolis, journal quotidien dont le tirage se monte à 20000 feuilles par jour, souhaite créer une mentalité journalistique différente, inconnue jusqu'alors en Grèce; les reportages, les interviews envoyés d'autres villes sont quelques-unes de nouveautés introduites par Acropolis. Le journal, se voulant populaire et n'ayant pas toujours une position politique 
stable, peut être considéré de tendance plutôt « conservatrice ». Les informations concernant le

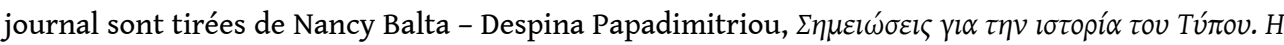

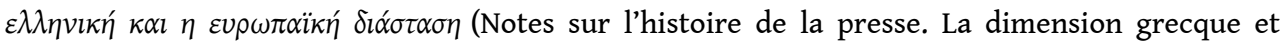
européenne), « Odysseas », Athènes, 1993, pp. 69-70.

12. Scrip était un journal d'orientation royaliste.

13. Embros, journal «populaire» et avec un profil nationaliste accentué, est un journal d'une grande diffusion.

14. Rizospastis, hebdomadaire d'orientation socialiste, paraît le 21/2/1908 à Thessalonique pour la première fois, par G. Filaretos. La propagation des idées socialistes par un grand nombre de revues dont les plus importantes la « Revue des Sciences Sociales et Juridiques » sous la direction d'Alexandre Papanastasiou, le journal « Socialisme » de P. Giannopoulos et A. Markellos, ainsi que le journal "Solidarité Ouvrière », organe de la Fédération de Thessalonique, crée l'ambiance idéale pour l'apparition d'un journal tel que Rizospastis. Ce journal, ouvertement antimonarchiste, est l'adversaire déclaré du journal Acropolis. En 1917 Rizospastis se déplace à Athènes, et, en 1921, il devient l'organe officiel du Parti Socialiste Ouvrier de Grèce (S.E.K.E). Voir, Balta Papadimitriou, op. cit. pp. 78-79.

15. Lambros Coromilas, Consul Général de la Grèce à Thessalonique est parmi les adversaires les plus durs du programme de Mürzsteg. Pour son raisonnement, qui exprime d'ailleurs les sentiments de la majorité du gouvernement grec, voir, Helen Gardikas - Katsiadakis, Greece and the Balkan Imbroglio: Greek Foreign Policy, 1911-1913, Athènes: Syllogos Pros Diadosin Ofelimon Vivlion, 1995, pp. 24-25.

16. Voir, Douglas Dakin, "The Greek Proposals for an Alliance with France and Great Britain, June - July 1907”, Balkan Studies, 3, 1962, pp. 43-60.

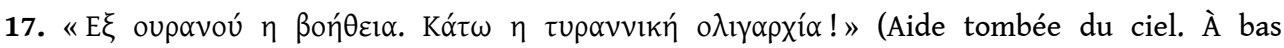
l'oligarchie tyrannique !), Rizospastis, 24, 18/31 juillet 1908.

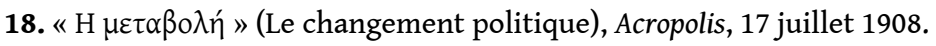


nouveau régime), Acropolis, 6/9/1908.

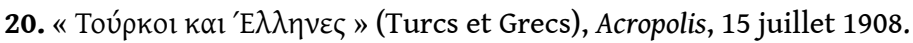

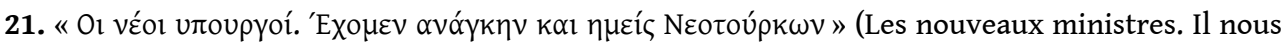
faut, à nous aussi, des Jeunes-Turcs), Acropolis, 18 juillet 1908.

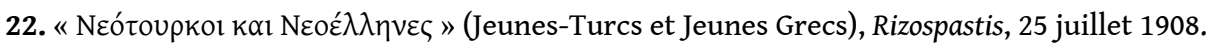

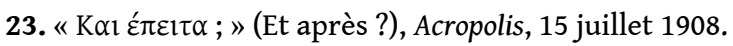

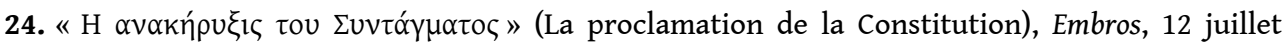
1908.

25. Par une série d'articles publiée dans Ecclésiastiki Alithia (désormais EA) - organe officiel du Patriarcat -, l'argumentation du cycle patriarcal contre la Constitution est explicitement

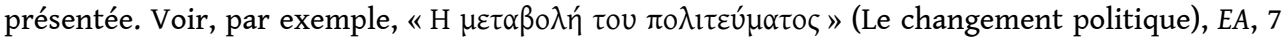

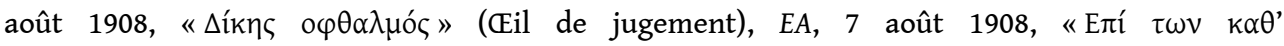
$\eta \mu \alpha ́$ ऽ » (Concernant les nôtres), EA, 12 septembre 1908, etc.

26. Acropolis, 18 juillet 1908.

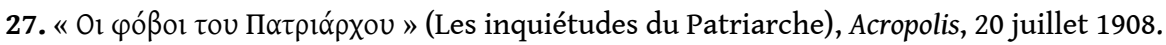

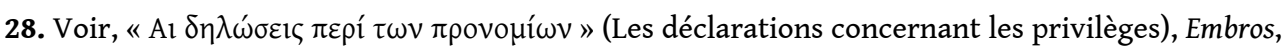

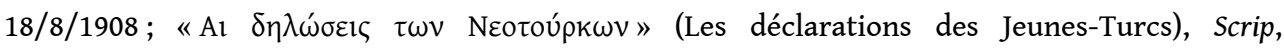
$18 / 8 / 1908$

29. Pour les privilèges du Patriarcat de Constantinople, ainsi que pour le processus de leur

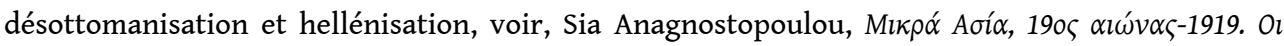

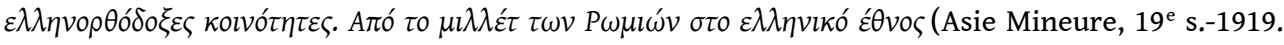


Les communautés grecques orthodoxes. Du millet de Rums à la nation grecque), Athènes : Ellinika Grammata, 1997, pp. 290-301, 428-438 et 461-467.

30. Extrait du discours prononcé par le député grec-orthodoxe au parlement ottoman, Charissios



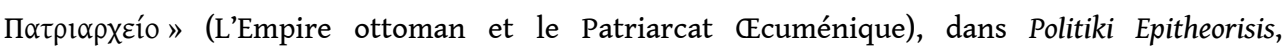
4 juillet 1910.

31. À titre indicatif, voir le raisonnement que le député Georges Boussios (présenté dans les minutes parlementaires sous le nom de Yorgi Boso) développe dans son discours prononcé au parlement ottoman dans le contexte de la discussion sur la Macédoine. Le député donc, après avoir parlé de l'unité traditionnelle, assurée pendant 600 ans par le sultan et le patriarche orthodoxe, conclut comme suit: "Ceux qui aspirent à la fragmentation de l'État ottoman aspirent à la fragmentation du Rumluluk [au sens de l'unité des orthodoxes] ». Meclisi Mebusan Zabit Ceridesi (désormais MMZC), d. 1, içt. sen. 1, 20. inikat, 19 Kanunusani 1324, pp. 407-408. [J'utilise la publication de 1980, faite par la maison de publication de la Grande Assemblée nationale].

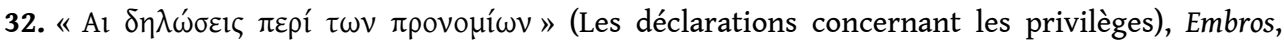
18 août 1908.

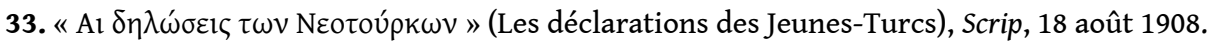

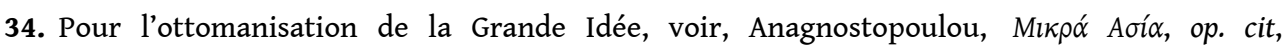
pp. 478-503.

35. Cette idée du turco-ottomanisme, bulgaro-ottomanisme, arméno-ottomanisme, etc., je la dois à Iléana Moroni, doctorante à l'EHESS, dont la thèse de doctorat portant sur les Jeunes-Turcs, est en voie de rédaction, sous la direction du professeur Maurice Aymard. D'après Karakisla d'ailleurs : « [...], il n'y avait pas de nation ottomane. Le Comité Union et Progrès s'est comporté comme si une nation ottomane existait. Cependant, ce que l'Union et Progrès entendait par nation ottomane c'étaient les Ottomans musulmans. [...]», Karakisla, Interview dans Istanbul Universitesi Siyasal Bilgiler Fakultesi Dergisi [Revue de la Faculté d'Études Politiques de l'Université d'Istanbul], no 38, mars 2008 (Numéro spécial sur la Révolution de 1908), pp. 7-30, p. 16.

36. Voir le discours au parlement ottoman du député Pancedoref Efendi (Monastir). MMZC, d. 1, ict. Sen. 1, 21. Inikat, 19 Kanunusani 1324, pp. 428-430.

37. Op. cit, p. 428.

38. MMZC, d. 1, ict. sen. 1, 17. inikat, Kanunusani 1324, c. 2, p. 393.

39. Voir, les discours des députés grec-orthodoxes Pavlos Carorilis (Smyrne) et Kozmidi Effendi (Istanbul) au parlement ottoman, MMZC, d. 1, ict. sen. 1, 17. inikat, Kanunusani 1324, c. 2, pp. 392-396.

40. MMZC, d. 1, ict. sen. 1, 20. inikat, 19 Kanunusani 1324, p. 408.

41. MMZC, d. 1, ict. sen. 1, 20. inikat, 17 Kanunusani 1324, p. 391.

42. Ibid.

43. Op. cit, p. 390.

44. «Dans le pays ottoman, la religion et la confession sont libres. Ceux qui le veulent ont des églises rum [orthodoxes patriarcales], ceux qui le veulent ont des églises bulgares ", explique au parlement le ministre de l'Intérieur. Huseyin Hilmi pasa. cf. MMZC, d. 1, ict. sen. 1, 20. inikat, 17 Kanunusani 1324, p. 368.

45. MMZC, d. 1, ict. sen. 1, 20. inikat, 17 Kanunusani 1324, p. 392.

46. Op. cit, p. 391.

47. Par l'article, sous le titre " Alt », le journal accuse les Grecs de Thessalonique, de Monastir et d'Istanbul, de fanatisme contre les Bulgares, et il souligne: "Nous devons être décents. L'Hellénisme en Turquie doit se comporter comme un peuple civilisé ». "Alt», Acropolis, 4 septembre 1908. 


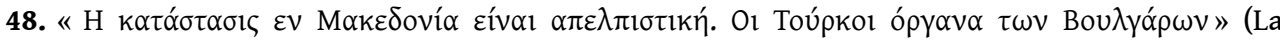
situation en Macédoine est désespérante. Les Turcs sont les organes des Bulgares), Acropolis, 5

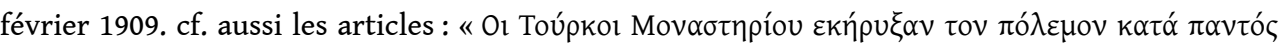
$\varepsilon \lambda \lambda \eta v i k o u$ » (Les Turcs du Monastir ont déclaré la guerre contre tout ce qui est grec), Acropolis, 1

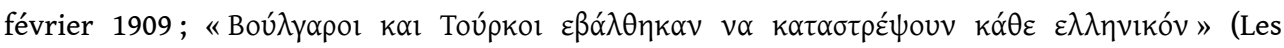
Bulgares et les Turcs ont entrepris à détruire tout ce qui est grec), Acropolis, 13 février 1909.

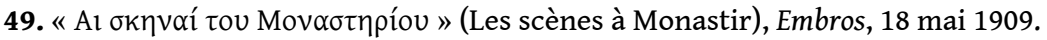

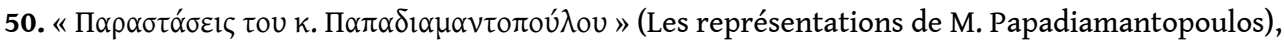
Scrip, 26 juin 1909.

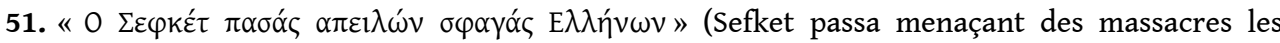
Grecs), Acropolis, 13 juin 1909.

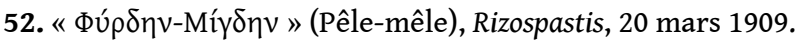

53. Pour les réformes de l'éducation visant à la centralisation du système éducatif, ainsi que pour

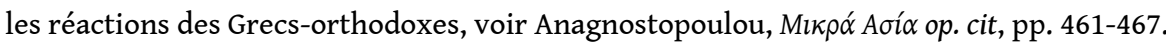

54. La question crétoise, une question épineuse pour le gouvernement grec, devient encore plus difficile lorsque, le 19 juin 1909, les Grecs de Crète remplacent les drapeaux ottomans et étrangers par des drapeaux grecs, violant par cet acte l'accord que le gouvernement grec avait fait avec le gouvernement ottoman. Ce dernier, pour lequel la question crétoise avait pris, par la presse ottomane interposée, l'ampleur d'une question nationale, applique immédiatement le boycott des produits grecs dans tous les ports ottomans. Pour la question crétoise intégrée dans



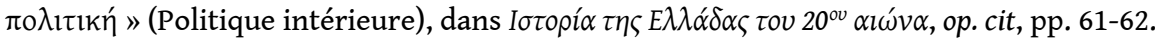

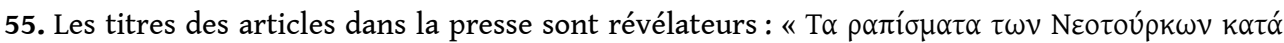

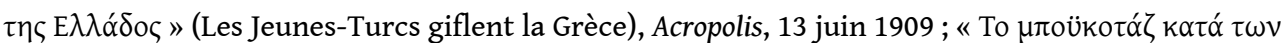

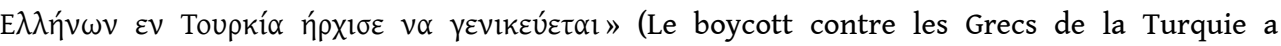

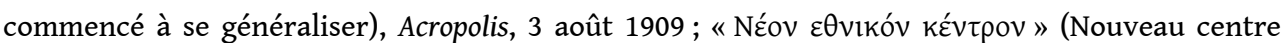
national), Rizospastis, 12 juin 1909.

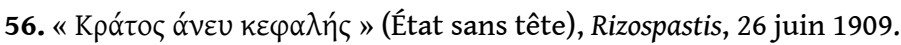

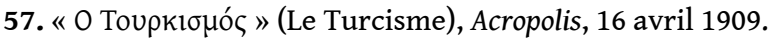

58. La lutte de bandes armées en Macédoine crée une ambiance favorable au renforcement du nationalisme parmi les hommes politiques. Le Parti nationaliste de Stephanos Dragoumis a commencé, à l'occasion de la révolte de Thérissos en Crète (1905), à lutter au sein du parlement grec pour une solution militaire des " problèmes nationaux » (Macédoine, Crète). Cette mentalité, de plus en plus dominante dans le Parlement, pousse le Premier ministre Théotokis de faire voter par le Parlement (1906-1909) une série de lois, envisageant de renforcer les forces armées grecques. Le but principal de la politique de Théotokis est de créer une force militaire, capable de faire la guerre sous le commandement d'un gouvernement national ; le but de la guerre serait la conquête des territoires ottomans. L'augmentation de l'armée grecque de 18.000 hommes en 1906 à 60.000 hommes en 1909 est indicative de la politique grecque. Pour une description détaillée des lois votées pour l'armement de l'Armée grecque, ainsi que pour la capacité de l'Armée en 1909, voir, Bohotis, op. cit, pp. 55-57.

59. Pour la militarisation de la société grecque, notamment de la petite bourgeoisie, voir Georges Mavrogordatos.

60. La ligue des corporations d'Athènes, qui jusqu'en 1906 était sous le contrôle des partis politiques, fait un accord en début août 1909 avec l'officier de liaison, par lequel elle déclare son soutien à sa politique. Par ailleurs, un assez grand nombre d'étudiants de l'Université d'Athènes créent « l'Union Universitaire » et déclarent, eux aussi, leur support à l'officier de liaison.

61. Pour le Patriarcat et ses privilèges pendant cette période et sous la pression d'Athènes, voir, Anagnostopoulou, Mikpá Aбía..., op. cit., pp. 476-486. 


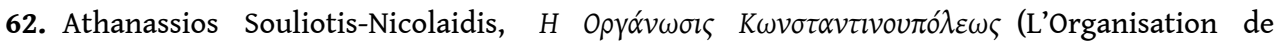
Constantinople), Athènes, pp. 125 et 127.

\section{RÉSUMÉS}

Entre juillet 1908 et le début de 1910, la presse grecque, face aux Jeunes-Turcs, passe de l'enthousiasme à la méfiance puis à la critique radicale. Elle espère d'abord que la liberté et l'égalité permettront l'helléno-ottomanisme, une domination gréco-turque de l'Empire qui éviterait l'emprise bulgare en Macédoine, sujet d'inquiétude principal des nationalistes grecs de 1908. En fait, le patriotisme "ottoman" est pour chaque "nation» de l'Empire une bonne occasion pour masquer son propre nationalisme. Au printemps 1909, la presse grecque découvre que l'ottomanisme peut laisser la place au "turcisme », son enthousiasme pour les Jeunes-Turcs tombe rapidement, le salut ne viendra pas de l'ottomanisme, mais de la Grèce, de sa préparation militaire face aux Turcs définitivement « barbares».

Between July 1908 and the beginning of 1910, the Greek press, towards the Young Turks, from enthusiasm changed to suspicion and radical criticism. Its first hopes are in a liberty and equality allowing to the helleno-ottomanism a greek-turkish domination which could push away any bulgarian hold over Macedonia, that is to say the main concern of Greek nationalists in 1908. In spring 1909, the Greek press understands that "helleno-ottomanism" may lead to "turkism", its enthusiasm for the Young Turks soon and quickly falls down, salvation won't come from "ottomanism" but from Greece itself and from its military preparation versus the Turks, for ever "barbarians".

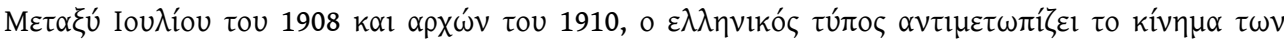

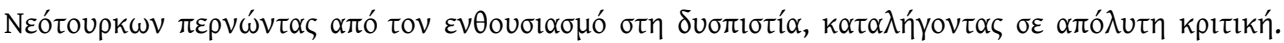

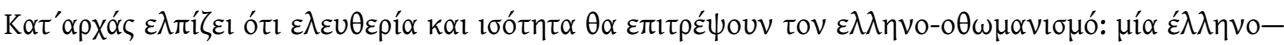

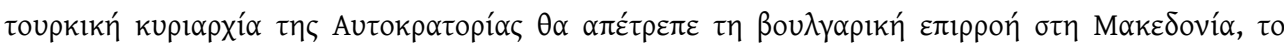

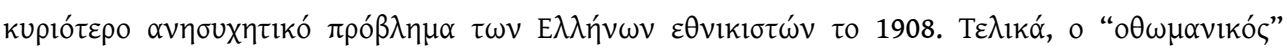

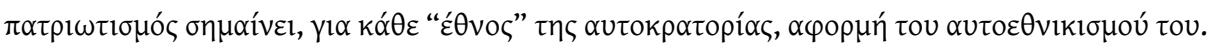

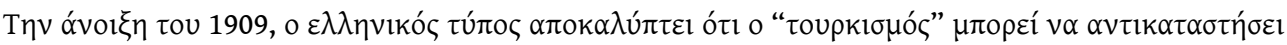

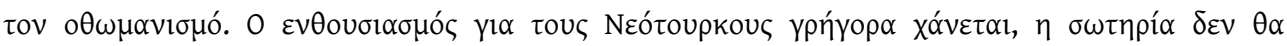

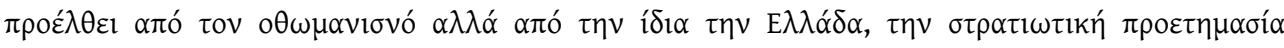

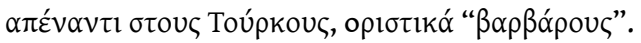




\section{INDEX}

Index géographique : Salonique, Athènes, Constantinople, Macédoine

Index chronologique : révolution jeune-turque (1908-1914), Empire ottoman

Thèmes : Histoire, Sciences politiques

motsclesmk АТИНА, МАКЕДОНИЈА, МЛАДОТУРСКАТА, ОТОМАНСКАТА ИМПЕРИЈА, СОЛУН,

ЦАРИГРАД

motsclestr Selanik, Makedonya, İstanbul, Atina, Genç Türkler, Osmanlı İmparatorluğu

Mots-clés : Acropolis, Embros, helléno-ottomanisme, Jeunes-Turcs, Helléno-ottomanisme, Dragoumis Ion (1878-1920), presse grecque, Giaour, question macédonienne, Goudi (mouvement révolution de), Grande Idée, Jeunes-Turcs, Sevket Pacha Mahmud (1856-1913), Millet,

Ottomanisation, Ottomanisme, Rizospastis, Rum/Roum, Scrip

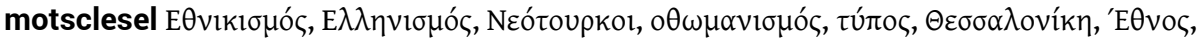

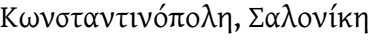

Keywords : helleno-ottomanism, macedonian question, Young Turks, Salonika, Macedonia, Constantinople, Athens, Politics, History, Young Turks revolution (1908-1914), Ottoman empire 